\title{
A Numerical Prediction of Tip Vortices from Tandem Propellers in the Counter-Rotating Type Tidal Stream Power Unit
}

\author{
Hoyun Jung*, Toshiaki Kanemoto, Pin Liu \\ Institute of Ocean Energy, Saga University, Saga, Japan \\ Email: *jung@ioes.saga-u.ac.jp
}

How to cite this paper: Jung, H., Kanemoto, T. and Liu, P. (2017) A Numerical Prediction of Tip Vortices from Tandem Propellers in the Counter-Rotating Type Tidal Stream Power Unit. Journal of Power and Energy Engineering, 5, 66-74. https://doi.org/10.4236/jpee.2017.512009

Received: November 8, 2017

Accepted: December 11, 2017

Published: December 14, 2017

Copyright ( 92017 by authors and Scientific Research Publishing Inc. This work is licensed under the Creative Commons Attribution International License (CC BY 4.0).

http://creativecommons.org/licenses/by/4.0/

\begin{abstract}
Ocean energy has a potential of providing a large amount of renewable energy around the world. One of the forms of ocean energy, tidal stream power is widely recognized as the continuous, predictable and eco-friendly ocean energy source. Unique tandem propellers that can counter-rotate have been designed to generate electric power effectively from a tidal stream. This type of power unit has several advantages compare to the conventional unit with a single propeller. At the design of the tidal stream power unit, it is important to investigate the structure of the tip vortex tubes shedding to predict the load of the propeller. In this research, we investigated the tip vortex shedding using the CFD method for the conventional single propeller and counter-rotating type tandem propellers and estimated the performance efficiency using RANS (Reynolds Averaged Navier-Stokes) model and we confirmed the limitation of RANS model on the calculation of the tip vortex stretching.
\end{abstract}

\section{Keywords}

Tidal Stream Power Unit, Counter-Rotation, CFD (Computational Fluid Dynamics), Tip Vortices, MRF (Multiple Reference Frame), Tandem Propellers

\section{Introduction}

Global warming is a big problem around the world. Due to an increasing demand in clean energy and rising fuel prices, more research is focused on renewable energy resources. Tidal stream energy is one type of renewable energy.

Among many renewable energy sources, tidal stream energy is continuous, predictable and eco-friendly ocean energy. Its energy density is higher than oth- 
er renewable energy resources, and it is easy to convert into other types of energy [1]. Tidal stream is caused by the flood and ebb of a tide due to the gravitational pull of the moon. The tidal stream power unit is placed in the path of tidal stream to generate electricity, which generates electricity by converting the horizontal tidal current flow into the rotational motion using propeller and generator.

Since the past one decade, many research efforts have been carried out to develop high-efficiency tidal current turbine. Now, various pilot test turbines have been developed and a number of projects are underway for commercialization in the coastal areas of UK and Europe, which have abundant tidal current energy resources.

A unique counter-rotating type tidal stream power unit is proposed by Kanemoto et al. [2] [3] [4] [5]. This tidal stream power unit has the tandem propellers and the peculiar synchronous generator with double rotational armatures. The schematic diagram of the counter-rotating type tidal stream power unit is shown in Figure 1. The front and the rear propellers counter-rotatesimultaneously the inner and the outer armatures respectively. This power unit has some benefits as shown below.

1) The induced voltage is about two times higher while keeping the propeller diameter the same as the conventional single propeller.

2) The propeller diameter can be reduced when keeping the induced the voltage same as the traditional one.

3) The cavitation can be suppressed through the relatively slower propeller rotating speeds.

4) The rotational moment is counter-balanced and the effect is low on the mounting system because the reaction force does not act on the outside, it is extremely easy to provide the unit with large capacity for not only the constructed power station with a mounting bet/pile but also the floating station moored at

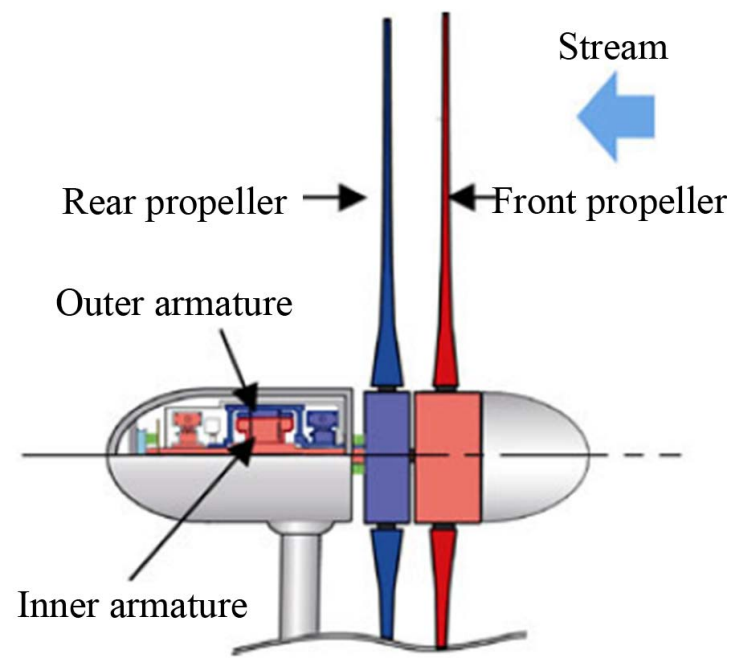

Figure 1. Schematic diagram of the counter-rotating type tidal stream power unit. 
the seabed with only one cable.

O'Regan et al. investigated vortex core size and trajectory using the LES model and URANS model. They confirmed that the LES models prediction was more accurate than the URANS model [7]. Clarke et al. represented the wake reduction and inter blade-spacing optimization for the contra-rotating turbine using CFD [8]. Morris et al. displayed the swirl characteristics for the various number of blades and estimation by swirl number [9]. Jo et al. checked the wake characteristics by the influence of the blade tip shape using the experimental and numerical method [10].

The purpose of this research is to investigate the performance of the single and the counter-rotating tidal stream power unit using the CFD method and to estimate the tip vortex structure using the RANS (Reynolds Averaged NavierStokes) model. The results were compared with the experimental results [6].

\section{Numerical Analysis}

We investigated the two types of models. The first one is the single propeller (using front blade) model and the second is the counter-rotating type propeller model.

Figure 2 shows the blade profiles of the front and the rear propellers and the sectional shapes between the blade hub to the blade tip. The front propeller is made up of 3 blades and the rear propeller consists of 5 blades. The diameter of front propeller is $500 \mathrm{~mm}$, and the rear one is $450 \mathrm{~mm}$. The size of the rear propeller is smaller than the front propeller. The counter-rotating type propellers have a torque balance between the front and rear propellers. So to increase the torque of the rear propellers, it is created by increasing the number of blades to 5.

Figure 3 represents the simulation region of the calculation. The flow domain is divided into 3 regions for the simulation of the single propeller. The boundary

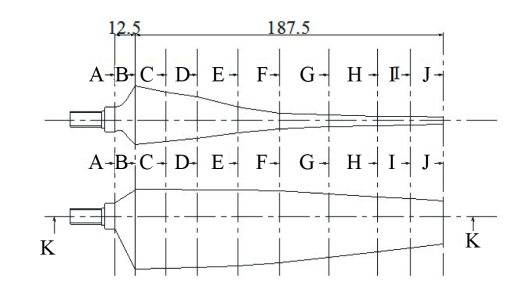

B-B D-D F-F F-F G-G H-H I-I J-J

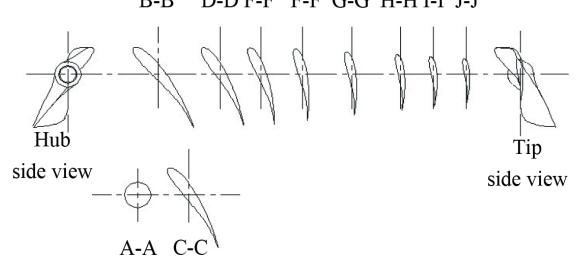

(a)

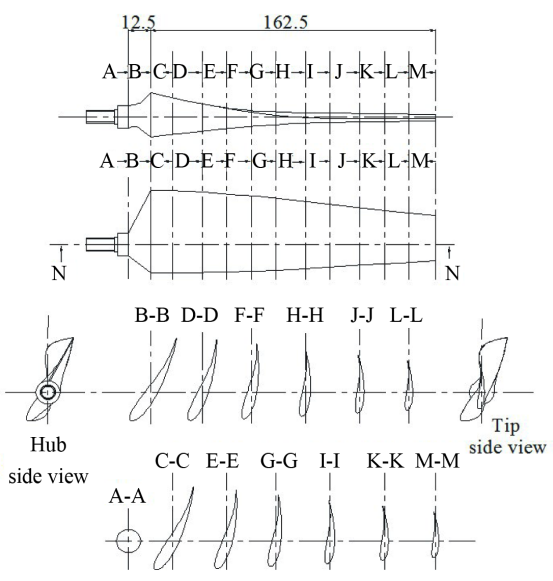

(b)

Figure 2. Blade sectional profiles of the counter-rotating type propellers. (a) Front propeller (3 blades); (b) Rear propeller (5 blades). 


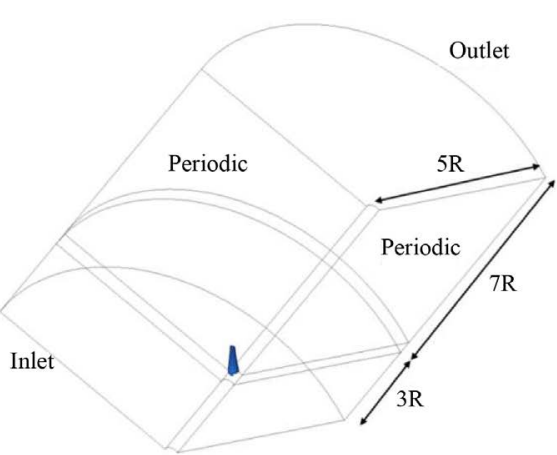

(a)

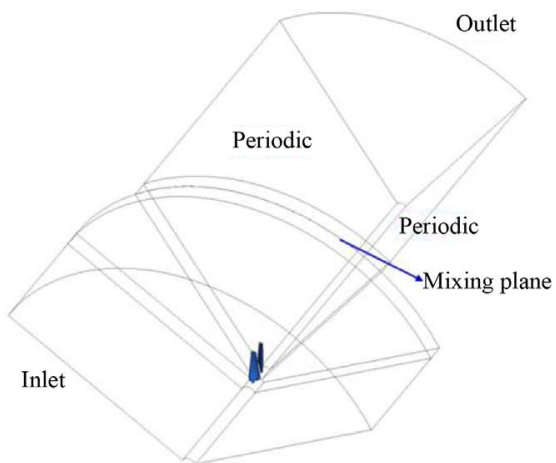

(b)

Figure 3. Numerical domains for the simulation. (a) Single propeller model; (b) Counter-Rotating type propeller model.

condition of the inlet is set as a velocity of $9.75(\mathrm{~m} / \mathrm{s})$. An outlet boundary has atmospheric pressure. The calculation for the same situation with the experimental status, we used air as a working fluid to compare to experimental results. The density of air is $1.225\left(\mathrm{~kg} / \mathrm{m}^{3}\right)$. The numerical domain size is set as $3 \mathrm{R}$ to upstream, 7R to downstream and $5 \mathrm{R}$ to radial direction. Periodic boundary condition is adopted to reduce the use of computing resources.

Figure $3(\mathrm{~b})$ shows the flow region of the counter-rotating propeller model. The flow passage angle for the periodic boundary condition including the front blade and the rear blade is different. Because there are 3 blades at the front propeller and 5 blades at the rear propeller. The angle of flow passage domain, front blade region has 120 degree and 72 degree of the rear blade region.

Figure 4 shows the grid system of the counter-rotating type propeller model for analysis. The hybrid grid system is used for the grid structure. By this method, it is possible to get rid of the difficulty of grid generation around complex geometries. Moreover, it can be more easily accommodated by simply changing the propeller region. It is used hexahedral mesh for the outer region and tetrahedral mesh and prism layer is constructed in the near blade region. The number of grids is 1,500,000 cells for the single propeller model and 3,000,000 cells for the counter-rotating type propellers. For all cases, we set the $y+$ value under 10.

The SST (Shear Stress Transport) turbulence model is adopted due to its known superior standards and its reliability to model flows having higher shear stress and to predict accurate results for flow separation under an adverse pressure gradient. This turbulence model uses a $k-\omega$ model for the near wall region and a $k-\varepsilon$ model for the bulk domain. To analyze the rotating effect of the flow fields, the MRF(Multiple Reference Frames) method is applied for the rotating problem. For the counter-rotating propeller model, mixing plane model is used for the non-conformal interface between the front and rear propeller region. The cell-centered finite volume method with a pressure-based velocity-pressure coupling algorithm was adopted. The solution gradients at the cell centers 


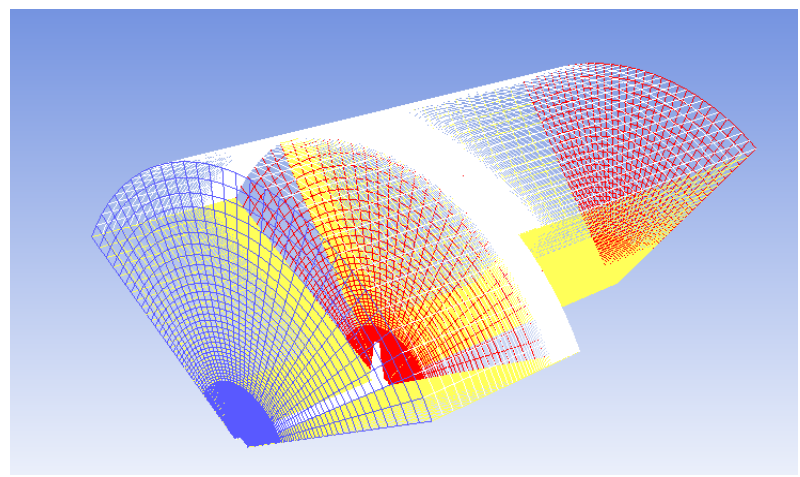

(a)

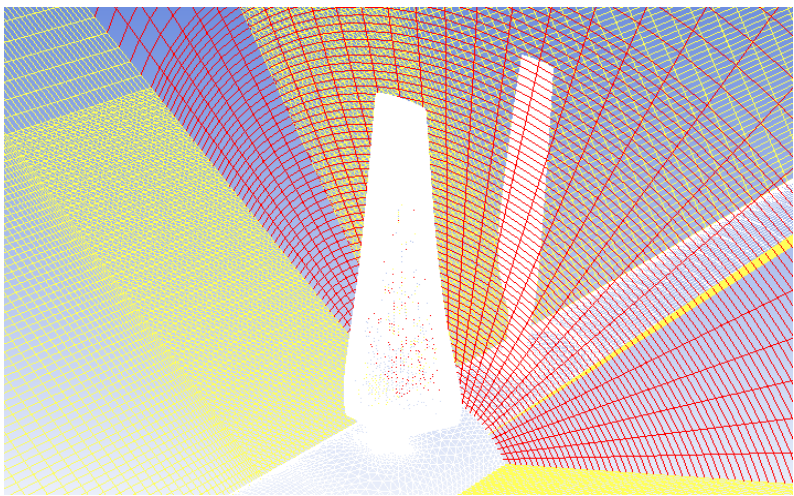

(b)

Figure 4. Grid structure for the calculation. (a) Grid shape for the counter-rotating type domain; (b) Grid shape of the near blades.

were evaluated by the cell center-based Green Gauss method. The convection term was discretized using the second order accurate upwind scheme, and for a diffusion terms, a central differencing scheme was used. The simulation was performed using ANSYS FLUENT V17.2, a commercial CFD software package [11].

To estimate the efficiency, we calculated the power coefficient related tip speed ratio. The equation is as follows.

$$
\begin{gathered}
\lambda_{f}=\frac{\omega_{f} R_{f}}{V_{i n}} \\
\lambda_{r}=\frac{\omega_{r} R_{r}}{V_{i n}} \\
\lambda=\lambda_{f}+\lambda_{r} \\
C_{P f}=\frac{T_{f} \omega_{f}}{(1 / 2) \rho V_{i n}^{3} A_{f}} \\
C_{P r}=\frac{T_{r} \omega_{r}}{(1 / 2) \rho V_{i n}^{3} A_{f}} \\
C_{P}=C_{P f}+C_{P r}
\end{gathered}
$$


where $\lambda$ is tip speed ratio, $\omega$ is rotating speed $[\mathrm{rad} / \mathrm{s}], R$ is radius of blade, $C_{P}$ is power coefficient, $T$ is torque $[\mathrm{Nm}], A$ is propeller swept area $\left[\mathrm{m}^{2}\right]$, $V_{\text {in }}$ is inlet wind velocity $[\mathrm{m} / \mathrm{s}], \rho$ is fluid density $\left[\mathrm{kg} / \mathrm{m}^{3}\right]$, subscript $f$ is front propeller, $r$ is rear propeller.

\section{Results and Discussion}

First an investigation on the efficiency of the propeller was conducted. Figure 5 shows the plot of the power coefficient $\left(C_{P}\right)$ variation along the TSR (tip speed ratio). The results of the simulation are compared to the experimental results. The circle symbols with line represent the results of the wind tunnel experiments. The counter-rotating type propeller has a higher efficiency than the single propeller and it is applicable for a wide range of tip speed ratio. The CFD results for the single propeller represent a good agreement with experimental results [3]. The maximum power coefficient value of the CFD analysis represents about 0.34 at the tip speed ratio of 3.5. At the calculation data of the counterrotating type propeller is shown as square symbol scatter. The Power coefficient values of the counter-rotating type propeller show a little different with the experimental results. But the tendency is nearly same. It may be caused the complex interaction at the interface between the front propeller region and rear propeller region. The maximum power coefficient value of the counter-rotating type propeller shows 0.45 at the tip speed ratio of 5.98 .

To examine the tip vortex shedding structure, vorticity contour is plotted in Figure 6. This figure represents vorticity distribution along the plane section to the downstream direction. We can confirm the vortex generation at the tip and near the trailing edge of the blade which has a strong value and the movement towards the downstream side. According to the flow is directed towards the downstream side, vorticity size is increased, but the strength is decreased. The radius of the vortex trajectory is unchanged along the flow movement towards the downstream side.

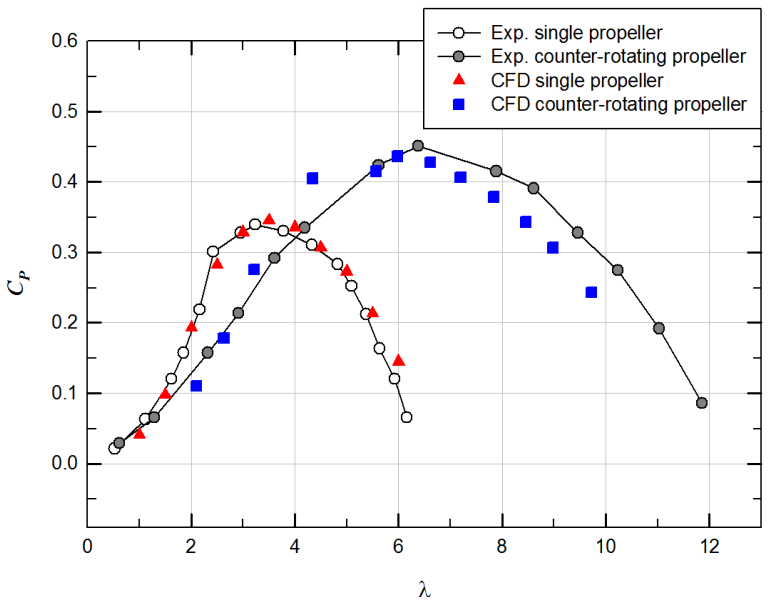

Figure 5. Power coefficient distributions according to the tip speed ratio. 
The 3 dimensional vortex core structures are shown in Figure 7 coloured by velocity. This figure shows the stretching of the tip vorticities. At the interface, we confirmed the discontinuities of wake (Figure 7(a)). We changed the simulation method to observe the vortex stretching. The simulation domain is divided
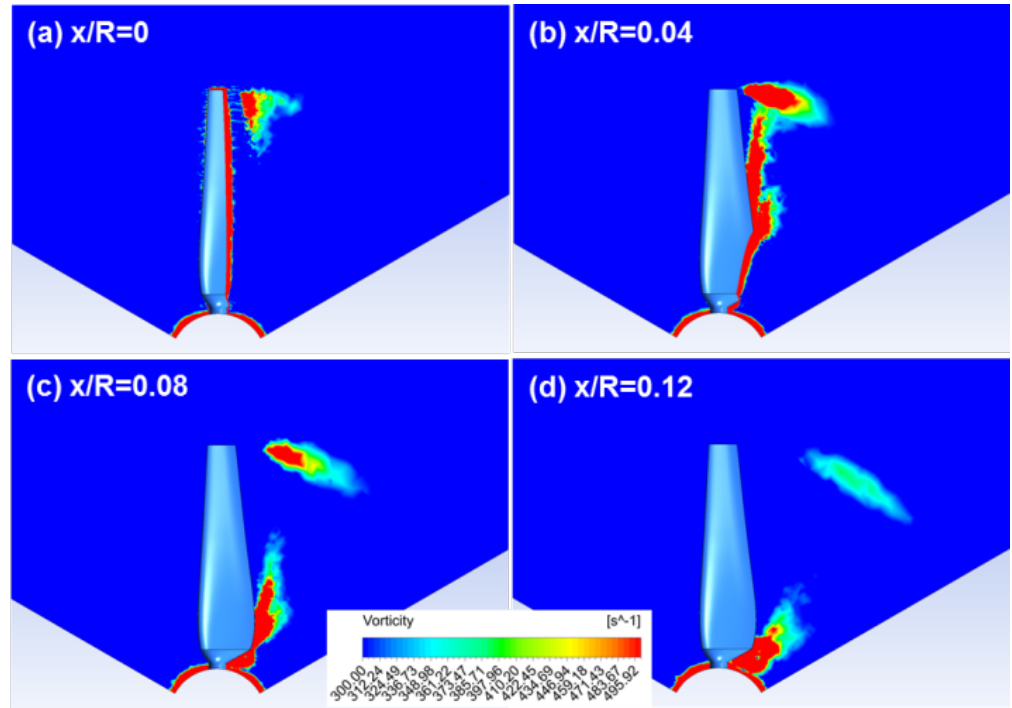

Figure 6. Vorticity contour according to the location of the downstream (at single propeller model).

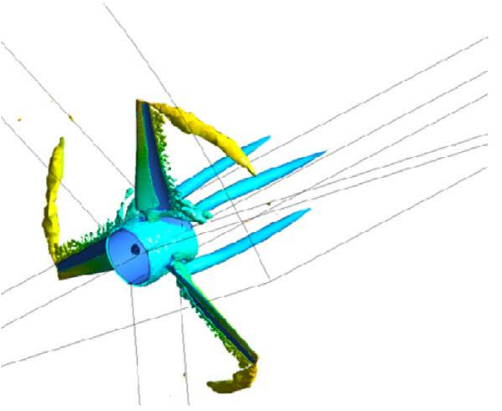

(a)

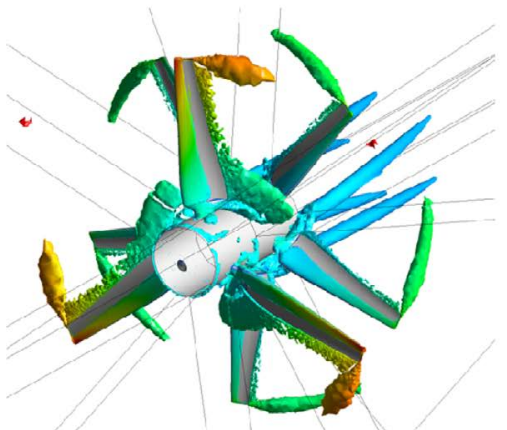

(c)

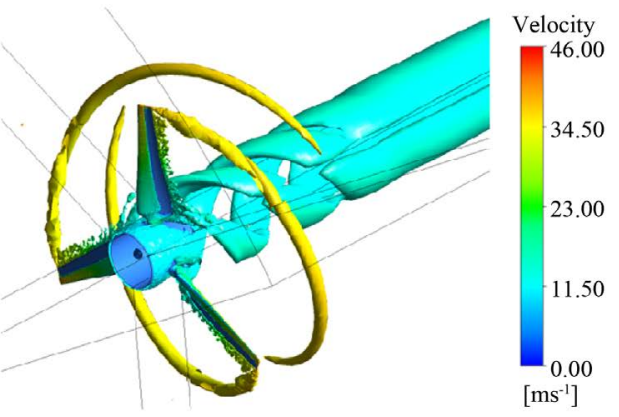

(b)

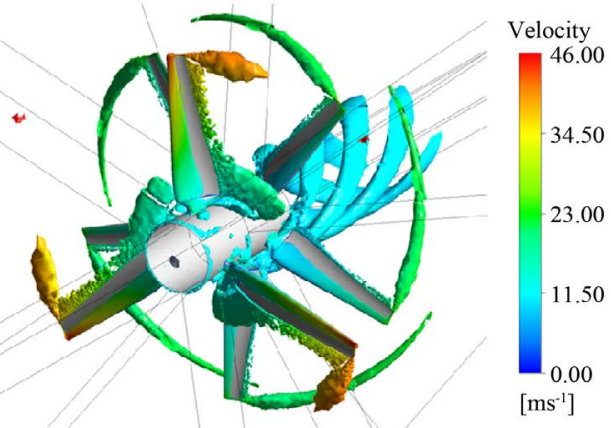

(d)

Figure 7. Vortex shedding structure around the blade at single and counter-rotating propeller model. (a) Stator state of rear region (single propeller); (b) Rotating state of rear region (single propeller); (c) Stator state of rear region (counter-rotating propeller); (d) Rotating state of rear region (counter-rotating propeller). 


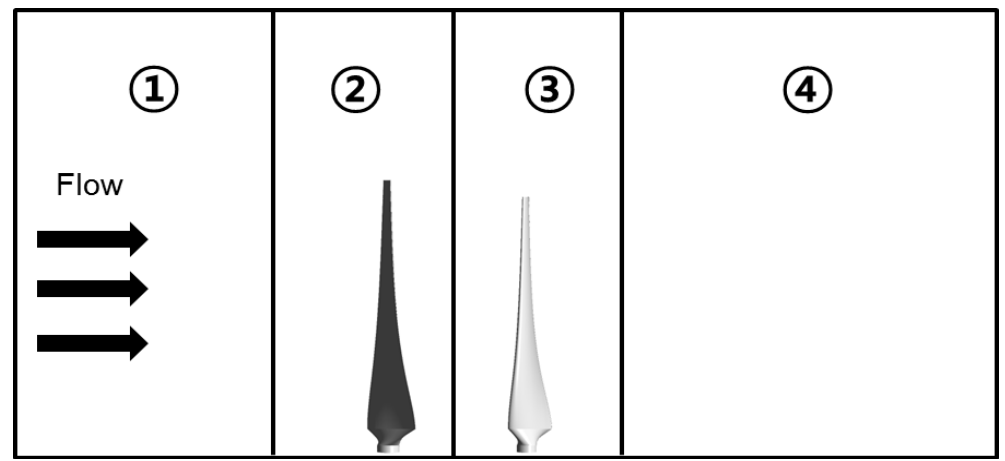

Figure 8. Divided region using interface surface for the simulation.

into 4 regions as shown in Figure 8. Region 1 is in stator state which means no rotating region. Region 2 and 3 is rotating region. And we changed the state of region 4 as a stator and rotating region. In the rotating case of region 4 , we can confirm the continuous vortex stretching.

\section{Conclusions}

In this study, the flow characteristics and the performance of the counter-rotating type tidal stream power unit using CFD method is numerically calculated. We predicted the tip vortices at downstream of the propellers by a RANS model. The results are summarized as shown below.

1) The power coefficient value is calculated by the CFD analysis. The results of the CFD have a good agreement with the experimental results. A maximum power coefficient of the single propeller model represents about 0.34 at TSR 3.5 and 0.45 at TSR 5.98 of the counter-rotating propeller model.

2) We can confirm the tip vortex stretching at downstream of the single and the counter-rotating propeller using a RANS model. But the tip vortices are disconnected at interface surface according to the changing the numerical method at the region 4. So, it is necessary to study other methods (e.g. URANS, Dynamic mesh or Overset grid method etc.) to obtain more accurate results.

\section{References}

[1] Bryans, A.G., Fox, B., Crossley, P. and Whittaker, T.J.T. (2004) Tidal Energy Resource Assessment for the Irish Grid. 39th International Universities Power Engineering Conference, Bristol, 6-8 September 2004, 1, 614-617.

[2] Kanemoto, T., Kaneko, M., Tanaka, D. and Yagi, T. (2000) Development of Counter-Rotating Type Machine for Water Power Generation (1st Report, Counter-Rotating Type Generator and Axial-Flow Runners). Transaction of the Japan Society of Mechanical Engineers, Series B, 66, 194-200. (In Japanese)

[3] Kanemoto, T., Tanaka, D., Kashwabara, T., Uno, M. and Nemoto, M. (2000) Counter-Rotating Type Machine Suitable for Tidal Current Power Generation. Proceedings of the Thenth International Offshore and Polar Engineering Conference, Seattle, 28 May-2 June 2000, 472-477.

[4] Huang, B. and Kanemoto, T. (2015) Performance and Internal Flow of a Counter-rotating Type Tidal Stream Turbine. Journal of Thermal Science, 24, 410-416. 
https://doi.org/10.1007/s11630-015-0803-1

[5] Wei, X.S., Huang, B., Liu, P. and Kanemoto, T. (2016) Performance Research of Counter-Rotating Tidal Stream Power Unit. International Journal of Fluid Machinery and Systems, 9, 129-136. https://doi.org/10.5293/IJFMS.2016.9.2.129

[6] Liu, P., Wei, X.-S., Heo, M.-W. and Kanemoto, T. (2016) Vortices Shedding from Blade Tips of Counter-Rotating Propellers Installed in Tidal Stream Power Unit. Proceedings of 6 th Asian Joint Workshop on Thermophysics and Fluid Science, Guilin China, 20-23 September 2016, (USB).

[7] O’Regan, M.S., Griffin, P.C. and Young, T.M. (2016) A Vorticity Confinement Model Applied to URANS and LES Simulations of a Wing-Tip Vortex in the Near-Field. International Journal of Heat and Fluid Flow, 61, 355-365.

https://doi.org/10.1016/j.ijheatfluidflow.2016.05.014

[8] Clarke, J.A., Connor, G., Grant, A.D., Johnstone, C.M. and Mackenzie, D. (2007) Development of a Contra-Rotating Tidal Current Turbine and Analysis of Performance. Proceedings of the 7 th European Wave and Tidal Energy Conference, Porto Portugal, 11-13 September 2007, 8 p.

[9] Morris, C.E., O’Doherty, D.M., Mason-Jones, A. and O’Doherty, T. (2016) Evaluation of the Swirl Characteristics of a Tidal Steram Turbine Wake. International Journal of Marine Energy, 14, 198-214. https://doi.org/10.1016/j.ijome.2015.08.001

[10] Jo, C.-H., Kim, D.-Y., Lee, K.-H., Rho, Y.-H. and Kim, K.-H. (2011) Design of Horizontal Axis Tidal Current Power Turbine with Wake Analysis. The Journal of the Korean Society for New and Renewable Energy, 9, 92-100. (In Korean) https://doi.org/10.7849/ksnre.2011.7.3.092

[11] ANSYS, Inc. (2016) ANSYS Fluent User's Guide, Release 17.2. 